of Serbia; ${ }^{4}$ nstitute of Biochemistry, Clinical Centre of Serbia; ${ }^{5}$ University Children's Hospital, Belgrade, Serbia

Background Diagnosis of perinatal hypoxic-ischemic encephalopathy (HIE) and early prediction neurological outcome is important and difficult. The aim of this study is to determine the value of neuron specific enolase (NSE) and lactate dehydrogenase (LDH) analysis in blood serum (BS) and cerebrospinal fluid (CSF) for evaluating severity HIE and predicting long term outcome in nenates with perinatal asphyxia.

Method 90 neonates (>32 weeks gestation) with perinatal HIE were enrolled prospectively. Perinatal HIE was categorised into three stages according Sarnat and Sarnat clinical scoring system and changes seen on amplitude integrated electroencephalography. NSE and $\mathrm{LDH}$ analysis in BS and CSF were taken during first 48h of age. Neurodevelopment outcome was assessed at 12 months of corrected gestational age using Denver Developmental Screening Test. Results Concentrations of NSE and LDH in CSF were significantly higher in neonates with advanced stage of HIE and corresponded well with subsequent neurodevelopment outcome $(p<0.01)$. Concentrations of $\mathrm{LDH}$ in $\mathrm{BS}$ were significantly higher in neonates with advanced stage of HIE and corresponded well with MODS $(p<0.01)$ and subsequent neurodevelopment outcome $(p<0.01)$ while concentrations of NSE in BP were no significantly higher in neonates with advanced stage of HIE ( $p>0.5)$.

Conclusions NSE and LDH analysis in CSF are accurate diagnostic tool for assessing extension of hypoxic-ischemic brain damage and early identification neonates with perinatal HIE who are at high risk of developmental delay. LDH analysis in BP also might offer an inexpensive, safe and simple prognostic tests for evaluating nenates with perinatal HIE

\section{USE OF SYNTHETIC COLLOIDS COMPARING NORMAL SALINE FOR NEURORESUSCITATION IN TERM NEWBORNS WITH SEVERE HIE}

doi:10.1136/archdischild-2012-302724.1102

D Surkov. NICU, Regional Children's Hospital, Dnipropetrovs'k, Ukraine

Background and Aims There is limited data according the use of $6 \%$ solution of hydroxyethyl starch $130 / 0.42$ in volemic resuscitation for neonates. The aim of the study was to compare the efficiency of $6 \%$ HES 130/0.42 and normal saline in the intensive care of term newborns with severe HIE.

Methods 15 full-term neonates with HIE were included. Score Sarnat II was in 9 newborns and Sarnat III was in 6 babies. The mean gestation age was $39.3 \pm 0.2$ weeks. Apgar score at $1^{\text {st }}$ minute was $3.8 \pm 0.1$ and at $5^{\text {th }}$ minute was $6.0 \pm 0.1$. Routine hemodynamic parameters, lab studies and cerebral perfusion pressure $(\mathrm{CPP}=1.1$ (Vs - Vd)/PI - 5 mm Hg) by transfontanel Doppler (Aaslid R., 1986) were collected at admission, in 1 hour and 3 hours after volume expanders infusion. The volume of normal saline was $20 \mathrm{ml} / \mathrm{kg}$ and of $6 \%$ HES $130 / 0.42$ was $10 \mathrm{ml} / \mathrm{kg}$. Results. Mean blood pressure at the 3 stages of study in response to $6 \%$ HES $130 / 0.42$ administration was 48-55-55 mm Hg, after normal saline 51-53-49 mm Hg. Cerebral perfusion pressure after administrarion of HES 130/0.42 was 7.7-11.8-15.7 mm Hg, after normal saline 14.1-17.6-17.1 mm Hg. Conclusions Use of HES 130/0.42 results in stable increasing of cerebral perfusion with normalizing of resistance index in front cerebral arteries. The effect occurs after administration of HES and remains up to 3-6 hours. Applying of HES 130/0.42 may be therapy of choice in low cerebral perfusion and/or hemodynamic instability in newborns with severe HIE.

\section{3 \\ NEW POSSIBILITY OF THE USE OF PREPARATION OF} LAZOLVAN AT PREMATURE INFANTS

doi:10.1136/archdischild-2012-302724.1103
MM Chuyko. The National Medical University of the name of Danylo Halytskiy, Lviv Ukraine

Introduction Ambroxol hydrochloride (drug Lazolvan) inherent anti-inflammatory and antioxidant effects that may have an impact onreducing the frequency and severity of intraventricular hemorrhage (IVH).

Purpose Installing the clinical efficacy of intravenous slow (15 min)administration of the drug Lazolvan (single dose $7.5 \mathrm{mg}$ twice a day from the first days of life for 7 days).

Methods In retrospective case-control study included 50 infants with gestational age less than 35 weeks. The main group $(n=18)$ received Lazolvan, control group not received. Before the beginning of the study formed the group did not differ among themselves on basic clinical and demographic indicators. Statistically significant group differ among themselves RDS third degree by the core group of children $-27.8 \%$ vs $6.26 \%$; $=0.03$. Clinical efficacy of the drug Lazolvan determined in terms of relative risk (RR) of IVH an indicator of patient who need to treat (NNT).

Results The relative risk of IVH in the main group 2.7 times less compared with the control group. At 2.17 times more often in terms of relative risk of IVH second degree occurred in the control group children. The treatment of 6.25 premature infants prevents a case of IVH. Newborn core group of perhaps less treated in the intensive care unit, respectively (days) $12.88 \pm 3.07$ vs $9.07 \pm 3.73$, $\mathrm{p}=0.0003$.

Conclusions The positive results from the use of Lazolvan in the complex treatmet of premature infants with risk of IVH prove its clinical effectiveness and extend its use.

\section{LONG-TERM OUTCOME OF TERM NEWBORNS WITH PERINATAL ASPHYXIA - PREDICTIVE FACTORS}

doi:10.1136/archdischild-2012-302724.1104

${ }^{1} \mathrm{~A}$ Avasiloaiei, ${ }^{2} \mathrm{C}$ Dimitriu, ${ }^{3} \mathrm{~A}$ Bivoleanu, 'L Paduraru, ${ }^{1} \mathrm{M}$ Stamatin. 'Neonatology; ${ }^{2}$ Biochemistry, Gr.T. Popa University of Medicine and Pharmacy; ${ }^{3}$ Neonatal Intensive Care Unit, Cuza-Voda Hospital of Obstetrics and Gynaecology, lasi, Romania

Perinatal asphyxia represents the second most important cause of death in the NICU and an important source of neurologic long-time sequelae.

Aim To identify long-term predictive factors for neurologic sequelae in term newborns with perinatal asphyxia.

Material and Methods We conducted a prospective study on 67 term newborns with perinatal asphyxia, admitted to our NICU between 2010-2011. The following parameters were followed: Apgar scores at 1, 5, 10 minutes, cord blood $\mathrm{pH}$, neurologic disorders, creatine-kinase (CK), lactate dehydrogenase ( $\mathrm{LDH})$, total antioxidant status (TAS) at 4, 12, 24, 48, 72 hours and 7 days, follow-up after discharge until 18 months of age.

Results Incidence of perinatal asphyxia in the NICU was $3.76 \%$. Mean Apgar score at 1 minute was 3.58 and at 5 minutes 5.33 , thus indicating the efficiency of resuscitation. Mean blood cord $\mathrm{pH}$ was 7.04. During the first 12 hours of life, all newborns had neurologic disorders. After the first 72 hours, this aspect was only present in $53.7 \%$ of the newborns. TAS was lowest at 12 hours (0.92 mmol/L), not reaching normal values at any moment. Survival was $91.04 \%$ and was correlated with ph $(p=0.012)$, CK $(p=0.04)$, LDH $(p=0.02)$, but not TAS $(p=0.063)$. Neurologic sequelae decreased progressively, reaching $10.45 \%(n=7)$ at 18 months. CK and LDH were predictive for sequelae ( $p=0.01)$, but not the Apgar score or TAS ( $p=0.08$ ).

Conclusion Routine determinations for blood cord $\mathrm{pH}, \mathrm{CK}$ and $\mathrm{LDH}$ can become valuable markers of long-term outcome for newborns with perinatal asphyxia, while TAS remains for further research. 


\section{OUTCOME AFTER INFLICTED TRAUMATIC BRAIN INJURY IN SHAKEN BABY SYNDROME: NEUROSURGICAL APPROACH}

doi:10.1136/archdischild-2012-302724.1105

F Menegazzo, M Bua, M Rosa Rizzotto, E Sgaravatti, P Facchin, R Faggin. Department of Pediatrics, Child Abuse Unit, University of Padova, Padova, Italy

Background and Aims Shaken Baby Syndrome (SBS) is a severe form of child abuse caused by violent shaking leading to severe head injuries, causing mild-severe long-term disabilities and death. The study aims to explore medium/long-term consequences of SBS comparing children undergoing neurosurgery with hematoma evacuation/cranioplasty with those with hematoma evacuation or no surgery.

Methods A cohort of 21 children with SBS, admitted to Pediatric Department/Padua Hospital (2003-2011), was followed-up. Each clinical record was reviewed collecting information on onset, acute course, ophthalmologic examinations, neuroimaging, treatment and procedures, family history and social background. Cases were followed-up at 3, 6 and 12 months after trauma, every year. The assessment included fundus evaluation and visual function, neuroradiological exams (MRI).

Results 21 cases were reviewed (M:F=2:1.1), 12 foreigners, 9 italians. Mean age at onset: 5.7 months (range 1.2-18). Mean age at last follow-up evaluation: 30.4 months (range 5-82). Mean follow-up duration: 24.6 months (range 1-73.5). In acute phase 8/19 underwent neurosurgical intervention and $1 / 21$ underwent eye surgery. 20/21 showed retinal hemorrhages, 21/21 cerebral hemorrhages, $11 / 21$ cerebellar hemorrhages, $5 / 10$ spinal subdural hematoma, 4/21 skull fractures, $6 / 21$ other body region fractures. At last follow-up evaluation resulted: $2 / 21$ hemiplegia, $1 / 21$ paraplegia, $1 / 21$ tetraplegia, $2 / 21$ cortical visual impairment, $3 / 21$ visual field deficits, 4/21 strabismus. 10/21 underwent cognitive and behavioural assessment demostrating in 6 cases global delay and in 4 cases delay in specific functions (locomotor, eye and hand coordination, performance scale). Surgical procedure's video will be shown.

Conclusions SBS may influence child development and therapeutical surgical approach seems crucial.

\section{IMPACT OF WARMED INHALED GAS FROM THE MECHANICAL VENTILATOR ON ESOPHAGEAL TEMPERATURE DURING WHOLE BODY HYPOTHERMIA FOR HYPOXIC-ISCHEMIC ENCEPHALOPATHY}

doi:10.1136/archdischild-2012-302724.1106

'S Sarkar, ${ }^{2}$ SS Sarkar, ${ }^{3}$ Bhagat, 'R Dechert, 'SM Donn. 'Department of Pediatrics, Division of Neonatal-Perinatal Medicine; ${ }^{2}$ Research Volunteer, Division of NeonatalPerinatal Medicine, University of Michigan Health System; ${ }^{P}$ Pediatrics, St Joseph Mercy Hospital, Ann Arbor, MI, USA

During whole body cooling (WBC), the core temperature is monitored with either an esophageal or a rectal probe. Most infants are usually on mechanical ventilation while receiving hypothermia. As the temperature in the esophagus responds rapidly to changes in the ambient temperature, inhalation of warmed gas from ventilator during hypothermia may lead to overestimation of ventilated patients' actual temperature, causing automated cooling devices to overcool patients well below set temperature targets.

Objective We determined if the esophageal temperature recordings during therapeutic WBC differ between ventilated and nonventilated infants.

Methods Twenty-two consecutively cooled infants had simultaneous esophageal and rectal temperatures recorded every 4 hours during 72 hours of WBC. The later was deemed to be actual core temperature. Other clinical monitoring and treatment during hypothermia were as per established protocol.
Results Fourteen infants received mechanical ventilation throughout cooling. The remaining 8 infants were on ventilator initially but got extubated and were not on ventilator during 32 to 72 hours section of WBC. Esophageal temperatures were significantly higher than simultaneous rectal temperatures ( $p \leq 0.01$ at each time point) for all 22 infants. However, the esophageal temperatures across every 4 hour time points during 32 to 72 hours section of WBC did not differ between the ventilated (n-14), and non-ventilated (n-8) infants. The magnitude (median, IOR) of the difference between esophageal and rectal temperatures were also similar between the 2 groups.

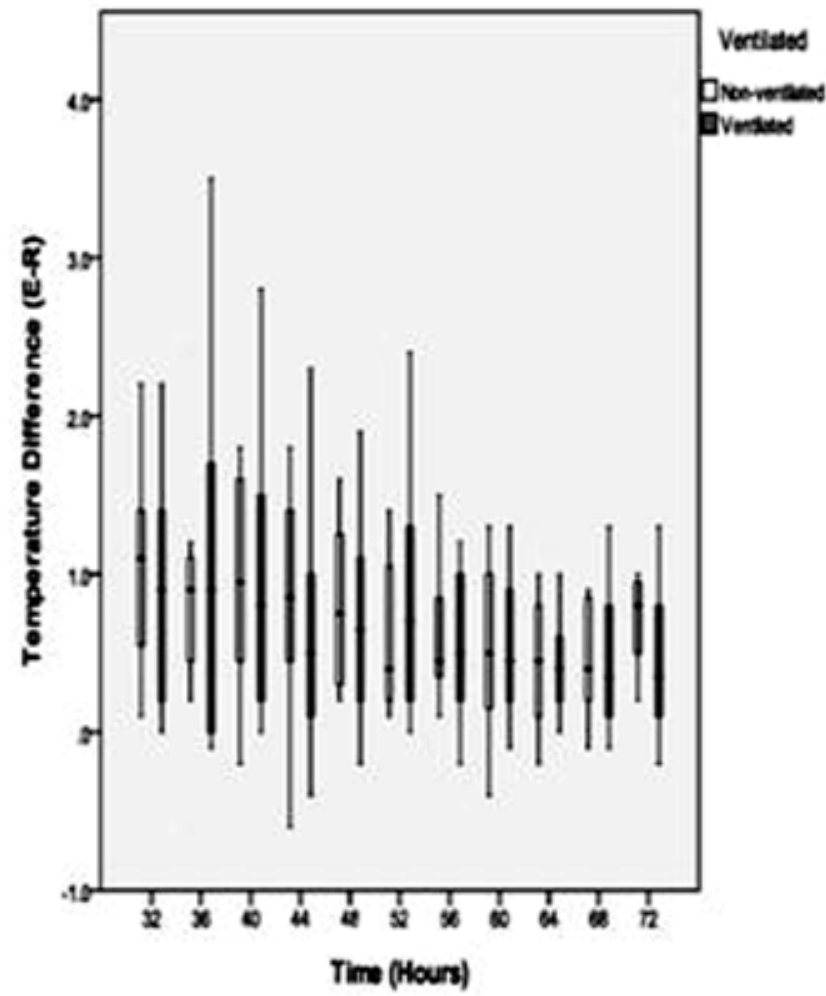

Abstract 1106 Figure 1 Comparison of E-R (in centigrade) between 2 groups

Conclusions Warmed inhaled gas does not interfere with the esophageal temperature during WBC.

\section{CONTINUOUS CARBON DIOXIDE MONITORING USING FEATURES OF NEONATAL ELECTROENCEPHALOGRAPHY}

doi:10.1136/archdischild-2012-302724.1107

${ }^{1} \mathrm{C}$ McKeering, ${ }^{1 P}$ Gaydecki, ${ }^{2} \mathrm{~A}$ Hendrickson, ${ }^{2} \mathrm{C}$ Jennings, $2,3 \mathrm{~S}$ Victor. ${ }^{1}$ School of Electrical and Electronic Engineering, University of Manchester; ${ }^{2}$ Newborn Intensive Care Unit, Central Manchester University Hospitals NHS Foundation Trust; ${ }^{3}$ School of Biomedicine, University of Manchester, Manchester, UK

Background The continuous monitoring of partial pressure of blood carbon dioxide $\left(\mathrm{pCO}_{2}\right)$ in premature babies has proven to be challenging. Spot measurements of $\mathrm{pCO}_{2}$ can be performed by taking a blood sample. However the frequency of such measurements is limited by their invasiveness.

Aim We aim to develop a continuous non-invasive method of predicting $\mathrm{pCO}_{2}$ using features of the preterm electroencephalography (EEG) signal.

Methods A regression model was trained on eight 12 hour EEG recordings that contained 22 blood gas measurements in total. All measurements were obtained from babies born before 28 weeks' gestation and less than 72 hours old. The duration of EEG quiescence 